\title{
Synthesis of new 4(3H)-quinazolinone derivatives under solvent-free conditions using PEG-400
}

\author{
Palle V. R. Acharyulu, ${ }^{a}$ P. K. Dubey, ${ }^{b^{*}}$ P. V. V. Prasada Reddy, ${ }^{c}$ and Thatipally Suresh ${ }^{c}$ \\ ${ }^{a}$ Dr. Reddy's Laboratories, Bachupalli, Hyderabad, India , 500072 \\ ${ }^{b}$ Department of Chemistry, College of Engineering., J N T University, Kukatpally, Hyderabad, \\ India, 500085 \\ ${ }^{c}$ Aptuit Laurus, Shamerpet, Thurakapally, Hyderabad, India, 500078 \\ E-mail: thatipally5@gmail.com
}

\begin{abstract}
Anthranilic acid 1 was reacted with chloroacetyl chloride in dichloromethane containing triethylamine to yield 2-chloromethyl benzo $[d][1,3]$ oxazin-4-one 2, which on treatment with aromatic amines yielded 2-(chloromethyl)-3-arylquinazolin-4(3H)-one 3. The latter on reaction with nitrogen nucleophiles such as ethyl piperidine-4-carboxylate, morpholine and piperidine-4one in $\mathrm{CH}_{3} \mathrm{CN}$ containing $\mathrm{K}_{2} \mathrm{CO}_{3}$ and catalytic amount of $\mathrm{KI}$ under refluxing conditions, gave 1(4-oxo-3-aryl-3,4-dihydroquinazolin-2-ylmethyl)piperidine-4-carboxylic acid ethyl ester (4), 2-morpholine-4-ylmethyl-3-aryl-3H-quinazolin-4-one (5), 2-(4-oxopiperidine-1-ylmethyl)-3aryl-3H-quinazolin-4-one (6) respectively. All the above reactions have been carried out under solvent-free conditions in the presence of PEG-400 by simple physical grinding in a mortar and pestle. The structures of all the new products obtained in the present work are supported by spectral and analytical data (IR, ${ }^{1} \mathrm{H}$ NMR and Mass spectroscopy).
\end{abstract}

Keywords: 2-(Chloromethyl)-3-arylquinazolin-4(3H)-one, nitrogen nucleophiles, solution phase, PEG-400, Solvent-free, mortar and pestle

\section{Introduction}

In recent years, considerable attention has been paid to reactions done under solvent-free conditions. ${ }^{1,2}$ One of the areas of central attention in this field includes reactions between solids. ${ }^{3,4}$ These reactions are not only of interest from an economical point of view, in many cases they also offer considerable synthetic advantages in terms of yield, selectivity and simplicity of the reaction procedure. 
Quinazoline-4(3H)-ones are versatile nitrogen heterocyclic compounds, displaying a broad spectrum of biological and pharmalogical activities such as anti-fungal, ${ }^{5}$ anti-tumour, ${ }^{6}$ hypotensive, ${ }^{7}$ anti-cancer, ${ }^{8,9}$ anti-HIV, ${ }^{10}$ anti-inflammatory, ${ }^{11}$ anti-bacterial ${ }^{12}$ etc. Furthermore, quinazoline-4(3H)-ones substituted at 2 and 3-position derivatives play a pivotal role in the hypotensive activity. ${ }^{13,14}$ Several bio-active natural products such as febrifugine (7) and isofebrifugine (8) contain quinazolinone moieties with potential anti-malarial activity.

Polyethylene glycol (PEG-400) ${ }^{15}$ has been applied here as an efficient reaction medium for the preparation of quinazolinone derivatives containing nitrogen cyclic ring systems. PEG-400 is a biologically acceptable inexpensive polymer and an eco-friendly reagent. The PEG-400 is widely used in many organic reactions for conversion ${ }^{16}$ of oxiranes to thiiranes, asymmetric aldol reactions ${ }^{17}$ in presence of L-proline, cross-coupling reaction, ${ }^{18}$ Baylis-Hillman reaction ${ }^{19}$ and ring opening of epoxides. ${ }^{20}$

We now wish to report here in synthesis of several newer derivatives of quinazolinones by incorporating different substituted aryl amines at 3-position and cyclic rings containing nitrogen nucleophiles such as ethyl-piperidine-4-carboxylate, morpholine and piperidine-4-one at 2position of quinazolinone nucleus in solution phase and also under solvent-free conditions using PEG-400 by simple physical grinding with mortar and pestle.<smiles>[R]C1CCCN[C@@H]1CC(=O)Cn1cnc2ccccc2c1=O</smiles>

$(+)$-febrifugine, $\mathrm{R}=\mathrm{OH}(7)$<smiles>O=c1c2ccccc2ncn1CC1(SO)O[C@H]2CCCN[C@H]2O1</smiles>

$(+)$-iso febrifugine $(\mathbf{8})$

\section{Results and Discussion}

Anthranilic acid (1) was treated with chloroacetyl chloride in presence of triethylamine (TEA) in dichloromethane at room temperature to obtain previously reported 2-chloromethyl benzo $[d][1,3]$ oxazin-4-one (2). ${ }^{21}$ The latter on treatment with m-anisidine in refluxing pyridine for 5-6 h, followed by simple processing resulted in the formation of 2-(chloromethyl)-3-(3methoxyphenyl) quinazolin-4(3H)-one $\mathbf{3} \mathbf{a}^{22}$ (i.e.3a, $\mathrm{Ar}=\mathrm{C}_{6} \mathrm{H}_{4}-3-\mathrm{OCH}_{3}$ ). The reaction of $\mathbf{3 a}$ with ethyl piperidine-4-carboxylate, $\mathrm{K}_{2} \mathrm{CO}_{3}$ and $\mathrm{KI}$, under refluxing acetonitrile for 90-120 min. resulted in the formation of 1-[3-(3-methoxy-phenyl)-4-oxo-3,4-dihydro-quinazolin-2-ylmethyl]piperidine-4-carboxylicacid ethyl ester 4a. Structure of the latter compound was established by spectral and analytical data. (Scheme 1, Table 1). 


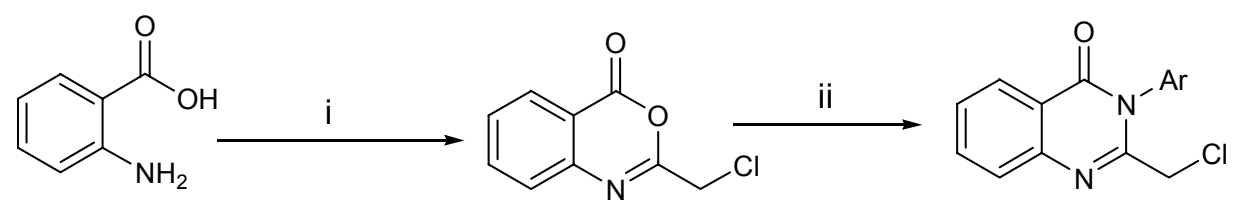

(1)

(2)

(3)

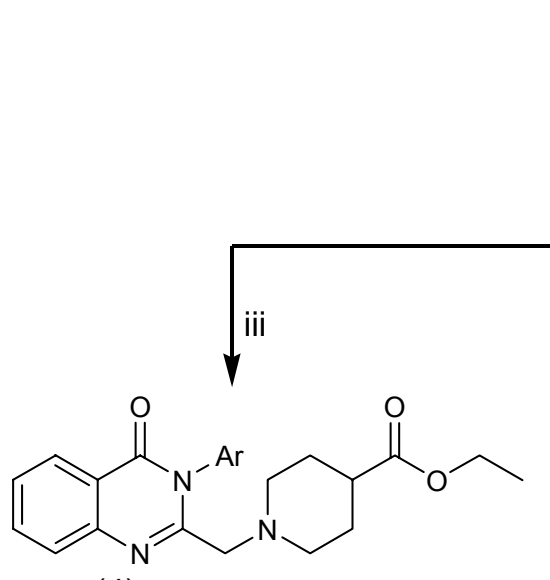

(4)<smiles>O=c1c2ccccc2nc(CCl)n1[Al]</smiles>

(3)

Scheme 1. Reagents and conditions: (i) Chloroacetylchloride, TEA, DCM; (ii) $\mathrm{Ar}-\mathrm{NH}_{2},(\mathrm{Ar}=\mathrm{a}$ $=-\mathrm{C}_{6} \mathrm{H}_{4}-3-\mathrm{OCH}_{3}, \mathrm{~b}=-\mathrm{C}_{6} \mathrm{H}_{5}, \mathrm{c}=-\mathrm{C}_{6} \mathrm{H}_{4}-2-\mathrm{CH}_{3} ; \mathrm{d}=-\mathrm{C}_{6} \mathrm{H}_{4}-2-\mathrm{CF}_{3}$, pyridine, $\Delta$, (iii) ethyl piperidine-4-carboxylate, $\mathrm{K}_{2} \mathrm{CO}_{3}, \mathrm{KI}, \mathrm{CH}_{3} \mathrm{CN}, \Delta$, (iv) morpholine, $\mathrm{K}_{2} \mathrm{CO}_{3}, \mathrm{KI}, \mathrm{CH}_{3} \mathrm{CN}, \Delta$ (v) piperidine-4-one, $\mathrm{K}_{2} \mathrm{CO}_{3}, \mathrm{KI}, \mathrm{CH}_{3} \mathrm{CN}, \Delta$. Under solvent-free conditions: iii/iv/v) ethyl piperidine-4-carboxylate / morpholine/ piperidine-4-one, $\mathrm{K}_{2} \mathrm{CO}_{3}, \mathrm{KI}, \mathrm{PEG}-400$, mortar and pestle.

Conversion of $\mathbf{3}$ to corresponding $\mathbf{4 , 5}$, and $\mathbf{6}$ derivatives is favored in presence of KI. This is probably due to the fact, that in presence of KI, chlorine of $\mathbf{3}$ is initially replaced by iodine, and subsequent reaction of iodo derivative of $\mathbf{3}$ with the nitrogen nucleophile is facile.

$$
\mathrm{R}-\mathrm{Cl}+\mathrm{KI} \rightleftharpoons \mathrm{R}-\mathrm{I}+\mathrm{KCl}
$$

\section{Scheme 2}

Alternatively, reaction of 3a with ethyl piperidine-4-carboxylate in presence of PEG-400 is carried out, in solid phase, by physical grinding in a mortar and pestle for $\sim 8 \mathrm{~min}$. (Table 1 , entry 3a) and subsequent work-up yielded product identical with $\mathbf{4 a}$, obtained above, in all respects characterized by comparison with mp, IR data.

It was found that above reactions between 3a and ethyl piperidine-4-carboxylate did not occur in the absence of PEG-400 even after grinding mixture of solids for 3-4 hrs. Thus it appears that PEG-400 acts like a Crown ether and that is why the addition of KI makes the reaction much faster because PEG-400 enhances the neucleophilicity of the iodide ion and facilitating reaction between $\mathbf{3 a}$ and ethyl piperidine-4-carboxylate. 
Table 1. Physical characterisation data for $4-6^{\mathrm{a}}$ compounds

\begin{tabular}{|c|c|c|c|c|c|c|c|c|}
\hline \multirow{2}{*}{$\begin{array}{l}\text { Starting } \\
\text { material }\end{array}$} & \multirow[t]{2}{*}{$\mathrm{Ar}$} & \multirow[t]{2}{*}{ Reagent } & \multirow{2}{*}{$\begin{array}{l}\text { Produ } \\
\text { ct }\end{array}$} & \multicolumn{2}{|c|}{ Solution phase } & \multicolumn{2}{|c|}{ Solvent-free } & \multirow[t]{2}{*}{ M.P $\left({ }^{\circ} \mathrm{C}\right)$} \\
\hline & & & & $\begin{array}{l}\text { Time } \\
(\min )\end{array}$ & $\begin{array}{l}\text { Yield } \\
(\%)\end{array}$ & $\begin{array}{l}\text { Time } \\
(\min )\end{array}$ & $\begin{array}{l}\text { Yield } \\
(\%)\end{array}$ & \\
\hline $3 \mathbf{a}$ & $\begin{array}{c}-\mathrm{C}_{6} \mathrm{H}_{4}-3- \\
\mathrm{OCH}_{3}\end{array}$ & & $4 a$ & 90 & 80 & 8 & 90 & (Liquid) \\
\hline $3 b$ & $-\mathrm{C}_{6} \mathrm{H}_{5}$ & & $4 b$ & 110 & 78 & 7 & 92 & (Liquid) \\
\hline $3 c$ & $-\mathrm{C}_{6} \mathrm{H}_{4}-2-\mathrm{CH}_{3}$ & & $4 c$ & 95 & 84 & 10 & 91 & (Liquid) \\
\hline 3d & $-\mathrm{C}_{6} \mathrm{H}_{4}-2-\mathrm{CF}_{3}$ & & $4 d$ & 120 & 78 & 12 & 89 & (Liquid) \\
\hline $3 \mathbf{a}$ & $\begin{array}{c}-\mathrm{C}_{6} \mathrm{H}_{4}-3- \\
\mathrm{OCH}_{3}\end{array}$ & & $5 \mathbf{a}$ & 95 & 86 & 7 & 95 & $130-32$ \\
\hline $3 b$ & $-\mathrm{C}_{6} \mathrm{H}_{5}$ & & $5 b$ & 105 & 80 & 8 & 94 & $146-50$ \\
\hline $3 \mathbf{c}$ & $-\mathrm{C}_{6} \mathrm{H}_{4}-2-\mathrm{CH}_{3}$ & & $5 c$ & 110 & 82 & 7 & 92 & $140-41$ \\
\hline 3d & $-\mathrm{C}_{6} \mathrm{H}_{4}-2-\mathrm{CF}_{3}$ & & $5 d$ & 115 & 76 & 10 & 90 & $165-66$ \\
\hline $3 \mathbf{a}$ & $\begin{array}{c}-\mathrm{C}_{6} \mathrm{H}_{4}-3- \\
\mathrm{OCH}_{3}\end{array}$ & & $6 a$ & 105 & 84 & 10 & 92 & $130-32$ \\
\hline $3 \mathbf{b}$ & $-\mathrm{C}_{6} \mathrm{H}_{5}$ & & $6 \mathbf{b}$ & 100 & 80 & 12 & 94 & $170-72$ \\
\hline $3 \mathbf{c}$ & $-\mathrm{C}_{6} \mathrm{H}_{4}-2-\mathrm{CH}_{3}$ & & $6 c$ & 110 & 80 & 12 & 90 & $158-60$ \\
\hline 3d & $-\mathrm{C}_{6} \mathrm{H}_{4}-2-\mathrm{CF}_{3}$ & & 6d & 120 & 75 & 15 & 88 & $168-70$ \\
\hline
\end{tabular}

${ }^{\mathrm{a}}$ Yields refers to products obtained after purification.
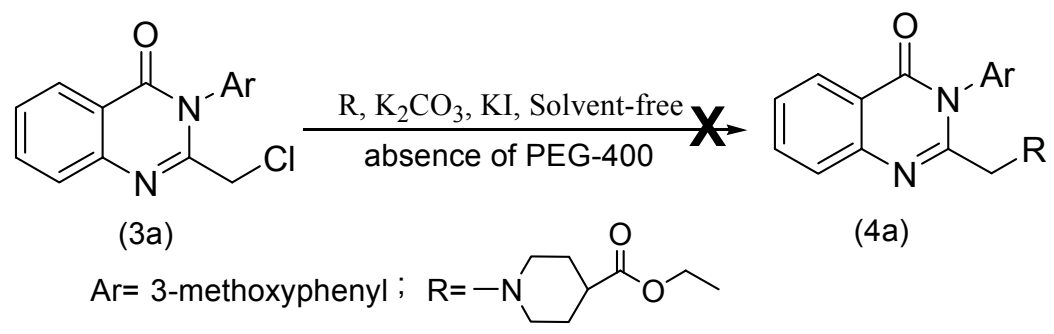

(4a)

Scheme 3 
Above reactions are of significant in nature when compare to the solution phase method in terms of time, yield and eco-friendly nature of the reaction.

Reaction between 3a and ethyl piperidine-4-carboxylate in solution phase and also under solvent-free conditions using PEG-400 as catalyst, has been found to be a general one and has been extended to other nitrogen nucleophilic substrates such as morpholine and pieridine-4-one and products thus obtained were assigned structures 5a and 6a respectively on the basis of their spectral data. Furthermore, reactions of ethyl piperidine-4-carboxylate, morpholine and pieridine-4-one are very general and have been found to occur with other $\mathbf{3}$ resulting in the formation of 4, 5, 6 (whose structures were assigned based on spectral data (Scheme 1, Table 1).

\section{Conclusions}

In summary, we have developed a simple and efficient method for preparation of new 4(3H)Quinazolinone derivatives in solution phase and also under solvent-free conditions using PEG400 by simple physical grinding in mortar and pestle at room temprature. Present protocol has several advantages, particularly solvent-free conditions, during work-up, water was used which is free from organic solvent (except product $\mathbf{4}$, which is liquid), fast reaction times, high yields, eco-friendly operational and experimental simplicity, readily available catalyst.

\section{Experimental Section}

General Procedures. Melting points were determined in open capillaries using Buchi melting point apparatus and are uncorrected. IR spectra were recorded on sample as diluted solutions (chloroform or carbon tetrachloride) in matched sodium chloride cells or as potassium bromide pellets with a Perkin IR spectrometer. ${ }^{1} \mathrm{H}$ NMR Spectra were recorded on a VARIAN $200 \mathrm{MHz}$ instrument with an internal standard of tetramethylsilane. Mass spectra were recorded on Agilent-LC-MS instrument giving only $\mathrm{M}^{+}$. values using $\left(\mathrm{M}^{+}+1\right)$ mode. Analytical TLC was performed with Silica gel GF-254from Merck \& Co., (Germany). Spots were detected with UVlight or in iodine. The following experimental procedures are representive of the general procedures used to synthesize all compounds.

\section{Preparation of $4 / 5 / 6$. General procedure in solution}

A mixture of 3 (10 mmol, 1.0 equiv), ethyl piperidine-4-carboxylate/morpholine/ piperidine -4one (10 mmol, 1.0 equiv), $\mathrm{K}_{2} \mathrm{CO}_{3}$ (2.76 g, $20 \mathrm{mmol}, 2.0$ equiv.), $\mathrm{KI}$ ( $0.57 \mathrm{~g}, 3 \mathrm{mmol}, 0.3$ equiv.) and $\mathrm{CH}_{3} \mathrm{CN}(20 \mathrm{~mL})$ was heated at $80{ }^{\circ} \mathrm{C}$ for $90-120 \mathrm{~min}$. The progress of reaction was monitored by TLC for complete disappearance of $\mathbf{3}$. On completion of reaction, mixture was diluted with water and extracted with ethyl acetate $(2 \times 25 \mathrm{~mL})$. The combined organic layer was 
washed with water, brine and then dried with anhydrous $\mathrm{Na}_{2} \mathrm{SO}_{4}$. The organic layer was distilled under reduced pressure, gave respectively 4/5/6. (Scheme 1, Table 1).

\section{Preparation of 4/5/6. General procedure under solvent-free}

A mixture of powdered anhydrous $\mathrm{K}_{2} \mathrm{CO}_{3}$ (4.14 g, 30 mmol, 3.0 eq), PEG-400 (10mol\%), KI (0. $57 \mathrm{~g}, 3 \mathrm{mmol}, 0.3$ equiv) and ethyl piperidine-4-carboxylate/morpholine/piperidine-4-one (10 mmol, 1.0equiv) were taken in a mortar and ground with a pestle for few minutes. To this mixture, starting material $\mathbf{3}(10 \mathrm{mmol})$ was added and the whole mixture was ground with pestle in the same mortar at room temperature for 7-15 $\mathrm{min}$. The progress of reaction was monitored by TLC. After complete disappearance of starting material, mixture was treated with ice-cold water $(50 \mathrm{ml})$. Product separated was filtered, washed with water, and dried to obtain 5/6 (Table-1). Product 4 separated out as oil, which was extracted in to DCM, washed with water, and organic layer on evaporation gave pure 4 as residue (Scheme 1, Table 1).

1-[3-(3-Methoxyphenyl)-4-oxo-3,4-dihydro-quinazolin-2-ylmethyl]piperidine-4-carboxylicacid ethyl ester (4a). IR ( $\mathrm{KBr}) \mathrm{cm}^{-1}: 1670,1730 .{ }^{1} \mathrm{H} \mathrm{NMR}\left(200 \mathrm{MHz}, \mathrm{CDCl}_{3}\right): \delta 1.2(\mathrm{t}, 3 \mathrm{H}$, $\mathrm{OCH}_{2}-\mathrm{CH}_{3}$ ), 1.5-2.6 (m, 9H, -piperidine), 3.1-3.3 (dd, 2H, $\left.-\mathrm{CH}_{2}\right), 3.8$ (s, 3H, $-\mathrm{OCH}_{3}$ ), 4.1 (q, $2 \mathrm{H}$, $\left.-\mathrm{OCH}_{2}-\mathrm{CH}_{3}\right), 6.85-8.3(8 \mathrm{H}, \mathrm{Ar}-\mathrm{H}) . \mathrm{M} / \mathrm{z}\left(\mathrm{M}^{+}+1\right)$ : 422 . Anal. Calcd. for $\left(\mathrm{C}_{24} \mathrm{H}_{27} \mathrm{~N}_{3} \mathrm{O}_{4}\right)$ requires: C, 68.39; H, 6.46; N, 9.97; Found: C, 68.34; H, 6.40; N, 9.92\%.

1-(-4-Oxo-3-phenyl-3,4-dihydro-quinazolin-2-ylmethyl]-piperidine-4-carboxylicacid ethyl ester (4b). IR ( $\mathrm{KBr}) \mathrm{cm}^{-1}: 1685,1728 .{ }^{1} \mathrm{H} \mathrm{NMR}\left(200 \mathrm{MHz}, \mathrm{CDCl}_{3}\right): \delta 1.2\left(\mathrm{t}, 3 \mathrm{H},-\mathrm{OCH}_{2}-\mathrm{CH}_{3}\right)$, 1.6-2.7 (m, 9H, piperidine), 3.2 (dd, 2H, $-\mathrm{CH}_{2}$ ), 4.1 (q, 2H, $-\mathrm{OCH}_{2}-\mathrm{CH}_{3}$ ), 7.3-8.3 (m, 9H, Ar-H). $\mathrm{M} / \mathrm{z}\left(\mathrm{M}^{+}+1\right)$ : 392. Anal. Calcd. for $\left(\mathrm{C}_{23} \mathrm{H}_{25} \mathrm{~N}_{3} \mathrm{O}_{3}\right)$ requires: $\mathrm{C}, 70.57 ; \mathrm{H}, 6.44 ; \mathrm{N}, 10.73$; Found: C, $70.51 ; \mathrm{H}, 6.38 ; \mathrm{N}, 10.75 \%$.

1-(-4-Oxo-3-o-tolyl-3,4-dihydro-quinazolin-2-ylmethyl]-piperidine-4-carboxylicacid ethyl ester (4c). IR (KBr) cm ${ }^{-1}: 1678,1730 .{ }^{1} \mathrm{H}$ NMR $\left(200 \mathrm{MHz}, \mathrm{CDCl}_{3}\right): \delta 1.2\left(\mathrm{t}, 3 \mathrm{H},-\mathrm{OCH}_{2}-\mathrm{CH}_{3}\right)$, 1.6-2.6 (m, 9H, piperidine), 2.2 (s, 3H, - $\left.\mathrm{CH}_{3}\right), 3.1-3.3$ (dd, $\left.2 \mathrm{H},-\mathrm{CH}_{2}\right), 4.1$ (q, $2 \mathrm{H},-\mathrm{OCH}_{2}-\mathrm{CH}_{3}$ ), 7.2-8.3 (m, 8H, Ar-H). M/z (M ++1$)$ : 406. Anal. Calcd. for $\left(\mathrm{C}_{24} \mathrm{H}_{27} \mathrm{~N}_{3} \mathrm{O}_{3}\right)$ requires: $\mathrm{C}, 71.09 ; \mathrm{H}$, $6.71 ; \mathrm{N}, 10.36$; Found: C, 71.00; H, 6.65; N, 10.28\%.

1-[4-Oxo-3-(2-trifluoromethyl-phenyl)-3,4-dihydro-quinazolin-2-ylmethyl]-piperidine-4carboxylicacid ethyl ester (4d). IR ( $\mathrm{KBr}) \mathrm{cm}^{-1}: 1680,1730 .{ }^{1} \mathrm{H} \mathrm{NMR}\left(200 \mathrm{MHz}, \mathrm{CDCl}_{3}\right): \delta 1.2$ (t, $\left.3 \mathrm{H},-\mathrm{OCH}_{2}-\mathrm{CH}_{3}\right), 1.6-2.6\left(\mathrm{~m}, 9 \mathrm{H}\right.$, piperidine), 3.15 (dd, $\left.2 \mathrm{H},-\mathrm{CH}_{2}\right), 4.1$ (q, 2H, $-\mathrm{OCH}_{2}-\mathrm{CH}_{3}$ ), 7.5-8.3 (m, 8H, Ar-H). M/z (M $\left.{ }^{+}+1\right)$ : 460. Anal. Calcd. for $\left(\mathrm{C}_{24} \mathrm{H}_{24} \mathrm{~F}_{3} \mathrm{~N}_{3} \mathrm{O}_{3}\right)$ requires: $\mathrm{C}, 62.74$; H, 5.26; N, 9.15; Found: C, 62.68; H, 5.20; N, 9.11\%.

3-(3-Methoxy-phenyl)-2-morpholin-4-ylmethyl-3H-quinazolin-4-one (5a). IR ( $\mathrm{KBr}) \mathrm{cm}^{-1}$ : 1672. ${ }^{1} \mathrm{H}$ NMR $\left(200 \mathrm{MHz}, \mathrm{CDCl}_{3}\right): \delta 2.3-2.4\left(\mathrm{~m}, 4 \mathrm{H}\right.$, morpholine), $3.2\left(\mathrm{~s}, 2 \mathrm{H},-\mathrm{CH}_{2}\right), 3.6(\mathrm{~m}, 4 \mathrm{H}$, morpholine), $3.9\left(\mathrm{~s}, 3 \mathrm{H},-\mathrm{OCH}_{3}\right), 6.9-8.3(\mathrm{~m}, 8 \mathrm{H}, \mathrm{Ar}-\mathrm{H}) . \mathrm{M} / \mathrm{z}\left(\mathrm{M}^{+}+1\right): 352$. Anal. Calcd. for $\left(\mathrm{C}_{20} \mathrm{H}_{21} \mathrm{~N}_{3} \mathrm{O}_{3}\right)$ requires: $\mathrm{C}, 68.36 ; \mathrm{H}, 6.02$, N, 11.96; Found: $\mathrm{C}, 68.32 ; \mathrm{H}, 6.08 ; \mathrm{N}, 11.98 \%$.

2-Morpholin-4-ylmethyl-3-phenyl-3H-quinazolin-4-one (5b). IR (KBr) cm ${ }^{-1}: 1676 .{ }^{1} \mathrm{H} \mathrm{NMR}$ $\left(200 \mathrm{MHz}, \mathrm{CDCl}_{3}\right): \delta 2.4\left(\mathrm{~m}, 4 \mathrm{H}\right.$, morpholine), 3.3 (s, 2H, $\left.-\mathrm{CH}_{2}\right), 3.5-3.6$ (m, 4H, morpholine), 
7.3-8.3 (m, 9H, Ar-H). $\mathrm{M} / \mathrm{z}\left(\mathrm{M}^{+}+1\right)$ : 322. Anal. Calcd. for $\left(\mathrm{C}_{19} \mathrm{H}_{19} \mathrm{~N}_{3} \mathrm{O}_{2}\right)$ requires: $\mathrm{C}, 71.01 ; \mathrm{H}$, 5.96; N, 13.08; Found: C, 71.05; H, 5.92; N, 13.02\%.

2-Morpholin-4-ylmethyl-3-o-tolyl-3H-quinazolin-4-one (5c). IR ( $\mathrm{KBr}) \mathrm{cm}^{-1}: 1684 .{ }^{1} \mathrm{H} \mathrm{NMR}$ $\left(200 \mathrm{MHz}, \mathrm{CDCl}_{3}\right): \delta 2.15\left(\mathrm{~s}, 3 \mathrm{H},-\mathrm{CH}_{3}\right), 2.2-2.4$ (m, 4H, morpholine), 3.1-3.3 (dd, 2H, $-\mathrm{CH}_{2}$ ), 3.5-3.6 (m, 4H, morpholine), 7.2-8.3 (m, 8H, Ar-H). $\mathrm{M} / \mathrm{z}\left(\mathrm{M}^{+}+1\right)$ : 336. Anal. Calcd. for $\left(\mathrm{C}_{20} \mathrm{H}_{21} \mathrm{~N}_{3} \mathrm{O}_{2}\right)$ requires: $\mathrm{C}, 71.62 ; \mathrm{H}, 6.31 ; \mathrm{N}, 12.53$; Found: $\mathrm{C}, 71.58 ; \mathrm{H}, 6.26 ; \mathrm{N}, 12.55 \%$.

2-Morpholin-4-ylmethyl-3-(2-trifluoromethyl-phenyl)-3H-quinazolin-4-one (5d). IR ( $\mathrm{KBr}$ )

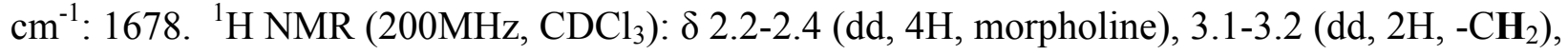
3.6 (q, 4H, morpholine), 7.5-8.3 (m, 8H, Ar-H). M/z (M + +1$)$ : 390. Anal. Calcd. for $\left(\mathrm{C}_{20} \mathrm{H}_{18} \mathrm{~F}_{3} \mathrm{~N}_{3} \mathrm{O}_{2}\right)$ requires: $\mathrm{C}, 61.69 ; \mathrm{H}, 4.66 ; \mathrm{N}, 10.79$; Found: $\mathrm{C}, 61.65 ; \mathrm{H}, 4.62 ; \mathrm{N}, 10.72 \%$.

3-(3-Methoxy-phenyl)-2-(4-oxo-piperidin-1-ylmethyl)-3H-quinazolin-4-one (6a). IR ( $\mathrm{KBr}$ ) $\mathrm{cm}^{-1}: 1670,1711 .{ }^{1} \mathrm{H}$ NMR $\left(200 \mathrm{MHz}, \mathrm{CDCl}_{3}\right): \delta$ 2.3. (m, 4H, piperidone), 2.6-2.8 (m, 4H, piperidone), 3.4 (s, 2H, $\left.-\mathrm{CH}_{2}\right), 3.8$ (s, 3H, $\left.-\mathrm{OCH}_{3}\right), 6.9-8.3(\mathrm{~m}, 8 \mathrm{H}, \mathrm{Ar}-\mathbf{H}) . \mathrm{M} / \mathrm{z}\left(\mathrm{M}^{+}+1\right): 364$. Anal. Calcd. for $\left(\mathrm{C}_{21} \mathrm{H}_{21} \mathrm{~N}_{3} \mathrm{O}_{3}\right)$ requires: C, 69.41, H, 5.82; N, 11.56; Found: C, 69.38; H, 5.80; N, $11.54 \%$.

2-(4-Oxo-piperidin-1-ylmethyl)-3-phenyl-3H-quinazolin-4-one (6b). IR ( $\mathrm{KBr}) \mathrm{cm}^{-1}$ : 1675 , 1714. ${ }^{1} \mathrm{H}$ NMR (200MHz, $\left.\mathrm{CDCl}_{3}\right): \delta 2.3(\mathrm{t}, 4 \mathrm{H}$, piperidone), $2.6(\mathrm{t}, 4 \mathrm{H}$, piperidone), $3.4(\mathrm{~s}, 2 \mathrm{H}$, $\left.\mathrm{CH}_{2}\right)$, 7.2-8.3 (m, 9H, Ar-H). $\mathrm{M} / \mathrm{z}\left(\mathrm{M}^{+}+1\right)$ : 334. Anal. Calcd. for $\left(\mathrm{C}_{20} \mathrm{H}_{19} \mathrm{~N}_{3} \mathrm{O}_{2}\right)$ requires: $\mathrm{C}$, 72.05; H, 5.74; N, 12.60; Found: C, 72.01; H, 5.78; N, 12.58\%.

2-(4-Oxo-piperidin-1-ylmethyl)-3-o-tolyl-3H-quinazolin-4-one (6c). IR (KBr) $\mathrm{cm}^{-1}: 1678$, 1714: ${ }^{1} \mathrm{H}$ NMR $\left(200 \mathrm{MHz}, \mathrm{CDCl}_{3}\right): \delta 2.2\left(\mathrm{~s}, 3 \mathrm{H},-\mathrm{CH}_{3}\right), 2.3(\mathrm{dd}, 4 \mathrm{H}$, piperidone), $2.6(\mathrm{dd}, 4 \mathrm{H}$, piperidone), 3.2-3.4 (dd, $\left.2 \mathrm{H},-\mathrm{CH}_{2}\right), 7.2-8.3(\mathrm{~m}, 8 \mathrm{H}, \mathrm{Ar}-\mathbf{H}) . \mathrm{M} / \mathrm{z}\left(\mathrm{M}^{+}+1\right): 348$. Anal. Calcd. for $\left(\mathrm{C}_{21} \mathrm{H}_{21} \mathrm{~N}_{3} \mathrm{O}_{2}\right)$ requires: $\mathrm{C}, 72.60, \mathrm{H}, 6.09, \mathrm{~N}, 12.10$; Found: $\mathrm{C}, 72.62 ; \mathrm{H}, 6.01 ; \mathrm{N}, 12.04 \%$.

2-(4-Oxo-piperidin-1-ylmethyl)-3-(2-trifluoromethyl-phenyl)-3H-quinazolin-4-one (6d). IR $(\mathrm{KBr}) \mathrm{cm}^{-1}: 1686,1717 .{ }^{1} \mathrm{H} \mathrm{NMR}\left(200 \mathrm{MHz}, \mathrm{CDCl}_{3}\right): \delta 2.3(\mathrm{~m}, 4 \mathrm{H}$, piperidone), 2.7-2.9 (m, 4H, piperidone) $3.3(\mathrm{dd}, 2 \mathrm{H}), 7.5-8.3(\mathrm{~m}, 8 \mathrm{H}, \mathrm{Ar}-\mathrm{H}) . \mathrm{M} / \mathrm{z}\left(\mathrm{M}^{+}+1\right): 402$. Anal. Calcd. for $\left(\mathrm{C}_{21} \mathrm{H}_{18} \mathrm{~F}_{3} \mathrm{~N}_{3} \mathrm{O}_{2}\right)$ requires: $\mathrm{C}, 62.84 ; \mathrm{H}, 4.52$, N, 10.47; Found: $\mathrm{C}, 62.80 ; \mathrm{H}, 4.46 ; \mathrm{N}, 10.42 \%$.

\section{Acknowledgements}

We are thankful to Dr. H. R. Mohan (Aptuit-Laurus) for his useful discussions in this work.

\section{References}

1. Merzer, J. D. Angew. Chem. Int Ed. 1998, 37, 2975.

2. Tanaka, T.; Toda, F. Chem. Rev. 2000, 100, 1025.

3. Toda, F. Acc. Chem. Res. 1995, 28, 480.

4. Khodaei, M. M.; Meybodi, F. A.; Rezai, F.; Salehi, P. Synth. Commun. 2001, 31, 2047. 
5. Ghorab, M. M. Farmco 2000, 55, 249.

6. Bradly, D. S. Tetrahedron Lett. 2001, 42, 1851.

7. Kumar, A.; Tyagi, M.; Shrivasthava, V. K. Indian. J. Chem. 2003, 42B, 2142.

8. EI-Brollosy, N. R.; Abdel-Megeed, M. F.; Genady, A. R. Alexandria J. Pharm. Sci. 2003, 17(1), 17.

9. Shab, B. R.; Bhatt, J. J.; Patel, H. H.; Undavia, N. K.; Trivedi, P. B.; Desai, N. C. Indian. J. Chem. 1995, 34B, 201.

10. Khili, M. A.; Soliman, R.; Furghuli, A. M.; Bekhit, A. A. Arch. Pharm. 1994, 327, 27.

11. Shivaram, H. B.; Padmaja, M. T.; Shivnanda, M. K.; Akbarali, P. M. Indian. J. Chem. 1998, $37 B, 715$.

12. Hess, H. J.; Cronin, T. H.; Scriabine, A. J. Med. Chem. 1968, 11, 140.

13. Aziza, M. A.; Nassar, M. W.; Abdel Hamide, S. G.; EI-Hakim, A. E.; EL-Azab, A. S. Indian. J. Heterocycl. Chem. 1996, 6(1), 25.

14. (a) Pandey, V. K.; Pathak, L. P.; Mishra, S. K. Indian. J. Chem. 2005, 44B, 2005, 1940. (b) Pattanaik, J. M.; Paranaik, M.; Bhatta, D. Indian. J. Chem. 1998, 37B, 1304.

15. Dickerson, T. J.; Reed, N. N.; Janda, K. D. Chem. Rev. 2002, 102, 3325.

16. Das, B.; Reddy, V. S.; Krishnaiah, M. Tetrahedron Lett. 2006, 47, 8471.

17. Chandrasekhar, S.; Reddy, N. R.; Sultana, S. S.; Narsihmulu, Ch.; Reddy, K. V. Tetrahedron 2006, 62, 338.

18. Li, J-H.; Hu, X-C, Liang, Y.; Xie, Ye-Xi. Tetrahedron 2006, 62, 31.

19. Chandrasekhar, S.; Narsihmulu, Ch.; Saritha, B.; Sulthana, S. S. Tetrahedron Lett. 2004, 45, 5865.

20. Das, B.; Krishnaiah, M.; Thirupathi, P.; Laxminarayana, K. Tetrahedron Lett. 2007, 48, 4263.

21. (a) Pandey, V. K.; Pathak, L. P.; Mishra, S. K. Indian. J. Chem. 2005, 44B, 1940. (b) Pattanaik, J. M.; Paranaik, M.; Bhatta, D. Indian. J. Chem. 1998, 37B, 1304. (c) Bergmann, E. D.; Barshai, R. J. Am. Chem. Soc. 1959, 81, 5641. (d) Fetter, J.; Czuppon, T.; Hornyak, G.; Feller, A. Tetrahedron 1991, 47, 9393.

22. Tani, J.; Yoshihisa Yamada, Y.; Toyonari Oine, T.; Ochiai, T.; Ishida, R.; Inoue, I. J. Med. Chem. 1979, 22, 95. 